\title{
Heterologous Microcompartment Assembly in Bacillaceae: Establishing the Components Necessary for Scaffold Formation
}

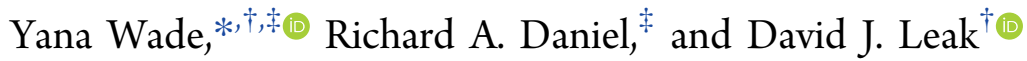 \\ ${ }^{\dagger}$ Department of Biology \& Biochemistry, University of Bath, Claverton Down, Bath, BA2 7AY, U.K. \\ ${ }^{\ddagger}$ Centre for Bacterial Cell Biology, Institute for Cell and Molecular Biosciences, University of Newcastle, Newcastle-upon-Tyne, NE2 \\ 4AX, U.K.
}

\section{Supporting Information}

ABSTRACT: Bacterial microcompartments (BMCs) are organelles that host specific biochemical reactions for both anabolic and catabolic functions. Engineered morphologically diverse BMCs bearing heterologous enzymatic pathways have shown enhanced productivity for commodity chemicals, which makes BMCs an important focus for metabolic engineering. Gaining control of $\mathrm{BMC}$ assembly and incorporation of a heterologous enzymatic cargo has yet to be achieved in thermophiles. Herein, we address this by first conducting a detailed bioinformatic analysis of the propanediol utilization $(p d u)$ operon in the thermophile Parageobacillus thermoglucosidasius. We then demonstrated, in vivo, the ability to assemble the native BMCs at an elevated temperature of $60{ }^{\circ} \mathrm{C}$. Heterologous expression of Pdu shell proteins from $P$. thermoglucosidasius in Bacillus subtilis resulted in the assembly of a single tubular BMC with an average length of $1.4 \mu \mathrm{m}$; BMCs assembled after a $20 \mathrm{~min}$ induction of expression of the shell operons. Moreover, we show that it is possible to target the monomeric superfolder GFP (msfGFP) to the interior of the compartment by fusion of an $\mathrm{N}$-terminal sequence of the propanediol utilization protein $(\mathrm{PduP})$ of at least 24 amino acids. This study establishes the feasibility of constructing cell factories for small molecules in industrially important Bacillus and Geobacillus spp. by heterologous cargo-carrying BMC production and assembly. Additionally, the study provides experimental confirmation that BMCs are produced in thermophilic bacteria, which opens a path for future research on repurposing the native organelles to provide new functionality at elevated temperatures.

KEYWORDS: bacterial microcompartments, shell, synthetic biology, biotechnology, thermophiles, Parageobacillus, Bacillus.
$\mathrm{B}$ acterial microcompartments (BMCs) are subcellular proteinaceous organelles hosting specific biochemical reactions for both anabolic and catabolic metabolism in a manner that is physically separated from other cytoplasmic process. ${ }^{1,2}$ They are found in a wide range of bacteria, being present in at least 23 phyla and incorporating 23 distinct types of metabolic functionality. Most of these functions have only been predicted from bioinformatic analysis and remain experimentally unverified. ${ }^{3}$

BMCs are divided into two distinct groups, carboxysomes and metabolosomes, based on their anabolic or catabolic functionality, respectively. Carboxysomes enclose $\mathrm{RuBisCO}$ and carbonic anhydrase together with elevated levels of carbon dioxide, resulting in enhanced autotrophic fixation of $\mathrm{CO}_{2}$ as part of the Calvin-Benson-Bassham cycle ${ }^{4,5}$ (Figure 1). They are present in all cyanobacteria and in some chemoautotrophic microorganisms and play an important role in global carbon fixation. ${ }^{5}$ The majority of the different types of BMCs are involved in catabolism. ${ }^{3}$ Metabolosomes can house multistep enzymatic pathways for conversion of metabolites such as propanediol, ${ }^{6}$ ethanolamine, ${ }^{7}$ choline, ${ }^{2}$ fucose, and rham- nose $\mathrm{e}^{8,9}$ into alcohols and acids via the production of highly reactive intermediates (Figure 1). These BMCs share a core biochemistry composed of a signature enzyme, which metabolizes a substrate into a corresponding aldehyde with the subsequent conversion into a phosphorylated product ( $\mathrm{R}$ P) by an aldehyde dehydrogenase and a phosphotransacylase or its alternative reduction to an alcohol by an alcohol dehydrogenase. An acyl kinase dephosphorylates the R-P product to an acid coupled with ATP production. Of the metabolosomes, the propanediol utilization (Pdu) system has been the most extensively characterized. The Pdu organelle contains a set of enzymes catalyzing the sequential breakdown of 1,2-propanediol (1,2-PD) into propionaldehyde by a signature enzyme, propanediol dehydratase, and its subsequent disproportionation into propanol and propionic acid. ${ }^{10}$

The shell of functionally diverse BMCs is assembled from proteins having a common topology that form hexamers (BMC-H), trimers (BMC-T), and pentamers (BMC-P)

Received: April 5, 2019

Published: June 26, 2019 


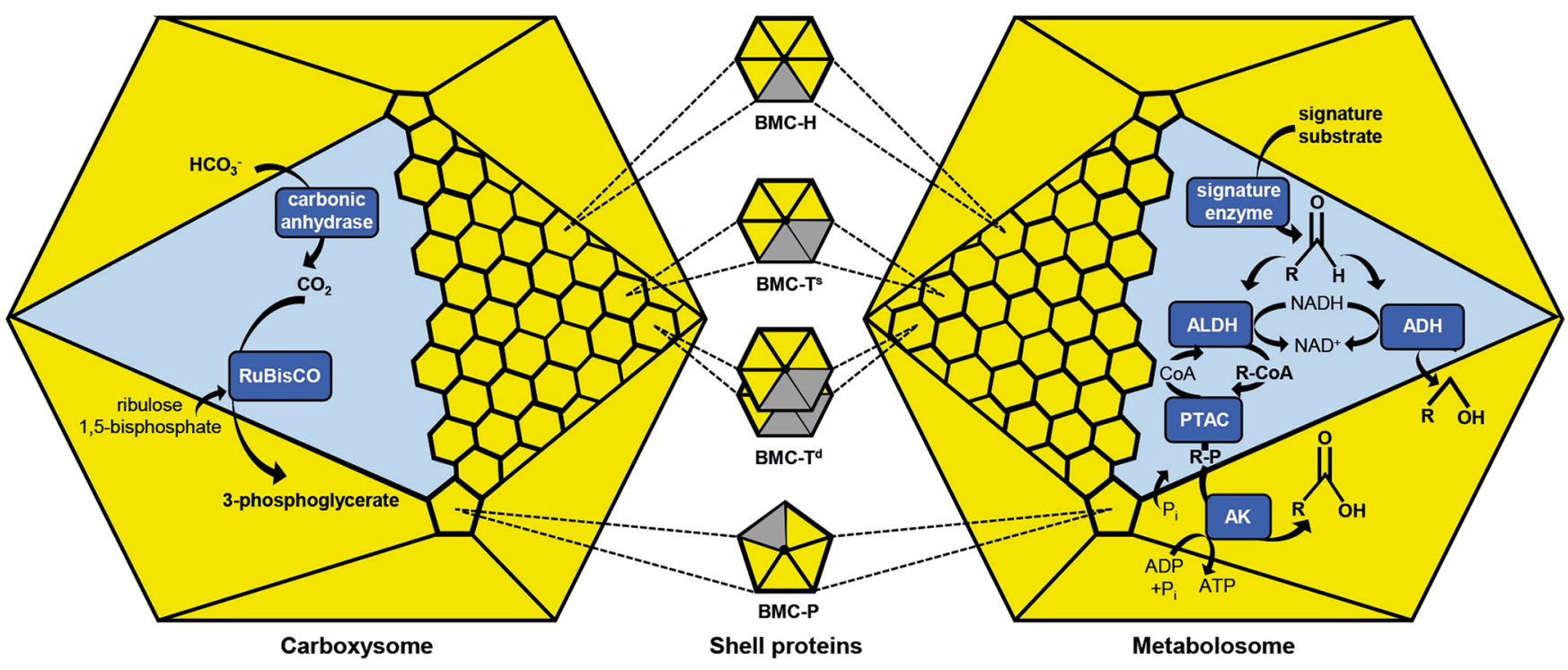

Figure 1. A simplified model of BMC structural and functional organization: ALDH, aldehyde dehydrogenase; ADH, alcohol dehydrogenase; PTAC, phosphotransacylase; AK, acyl kinase.

(Figure 1). BMC-H contains a single conserved BMC domain (Pfam00936) having one convex and one concave side, and is a highly abundant component of the BMC shell. BMC-T proteins are morphologically similar to $\mathrm{BMC}-\mathrm{H}$, but form trimers consisting of two tandemly fused Pfam00936 domains and are, thus, termed pseudohexamers. They are subdivided into two protein types: $\mathrm{BMC}^{\mathrm{S}} \mathrm{T}^{\mathrm{s}}$, which forms a single trimer, and $\mathrm{BMC}-\mathrm{T}^{\mathrm{d}}$ that contains two trimers, dimerized across their concave faces. BMC-P contains a single Pfam03319 domain, which forms cyclic pentamers. The faces of the organelle are assembled from a combination of BMC-H and BMC-T proteins, while the vertices of the polyhedral shell are capped by BMC-P proteins. ${ }^{11}$ The concave side of the shell proteins faces outward in recombinant BMC structures, ${ }^{12}$ whereas strong evidence supports a counter orientation, concave inward, in native systems. ${ }^{13,14}$ With tightly packed shell proteins, the pores at the cyclic symmetry axes of hexamers and pseudohexamers serve as a transport channel for the exchange of metabolites between the lumen of organelle and the cytosol. ${ }^{15}$ Although carboxysomes are usually found to have an icosahedral geometry, metabolosomes typically have more irregular polyhedrons. The observed size range of BMCs is $40-600 \mathrm{~nm} .^{16}$

It is thought that the function of a BMC is to enhance the metabolic pathway flux by enzyme colocalization and concentration, accumulation of volatile compounds such as $\mathrm{CO}_{2}$, and toxicity mitigation of produced intermediates such as propionaldehyde. ${ }^{17,18}$ The construction of a synthetic pyruvate-to-ethanol organelle, using an alcohol dehydrogenase (AdhB) and a pyruvate decarboxylase (Pdc) from Zymomonas mobilis and the Pdu shell of $C$. freundii, resulted in the synthesis of an elevated amount of ethanol in E. coli compared to enzymes that lacked BMC encapsulation. ${ }^{19}$ Recently, a cytotoxic lysis protein E was encapsulated inside the Pdu shell to restrict its toxic effect on the E. coli host. ${ }^{20}$ Thus, the redesign of native BMCs for the purpose of establishing nanobioreactors inside bacterial cells has great potential for biotechnology.

Besides being a source of a vast range of thermostable and thermoactive biomolecules including lipases, proteases, glyco- side hydrolases, and DNA polymerase I, Geobacillus spp. are of biotechnological and industrial importance as producers of ethanol and isobutyl alcohol from renewable resources. ${ }^{21}$ The genus Geobacillus was taxonomically separated from the obligate thermophilic Bacillus group $5,^{22}$ which was subsequently divided into Geobacillus and Parageobacillus, according to bioinformatic analysis of their full genomes. ${ }^{23}$

Bacillus species are well-known cell factories for robust, efficient, and cost-effective production of numerous industrial, biotechnological, and pharmaceutical proteins, vitamins, antibiotics, nucleosides, and biochemicals. ${ }^{24}$ The nonpathogenic and exo- and endotoxin-free, heterologous Bacillus spp. hosts have been generally regarded as safe for use in the food and drug industries. Significant fundamental knowledge and progress in genetic manipulation of Bacillus spp. has consolidated its position as one of the most important chassis organism for application for industrial microbiology. ${ }^{25}$

The ability to assemble BMCs in mesophilic and thermophilic candidate-industrial hosts from the Bacillaceae family would increase the potential for small molecule synthesis in this catabolically versatile group of organisms, particularly where the production of toxic or volatile intermediates is involved. To the best of our knowledge, empirical studies on in vivo BMC production have not previously been conducted at elevated temperatures or used components derived from thermophilic bacteria. In this work, we focus on the induction of a native $p d u$ operon in the thermophile, P. thermoglucosidasius NCIMB 11955 at $60{ }^{\circ} \mathrm{C}$ and production of empty BMCs derived from this system together with encapsulation of non-native cargo in the mesophilic model organism, B. subtilis.

\section{RESULTS}

Shell Proteins Are Widely Distributed in Parageobacillus spp. Despite the metabolically diverse roles of BMCs, due to variation in their encapsulated core enzymes and wide phylogenetic range in the bacteria possessing them, the homologous shell proteins share two highly conserved domains, the BMC 00936 (pfam00936) and EutN/CcmL 03319 (pfam03319). ${ }^{16,26,27} \mathrm{~A}$ search for both the BMC and 
(a) P. thermoglucosidasius NCIMB 11955

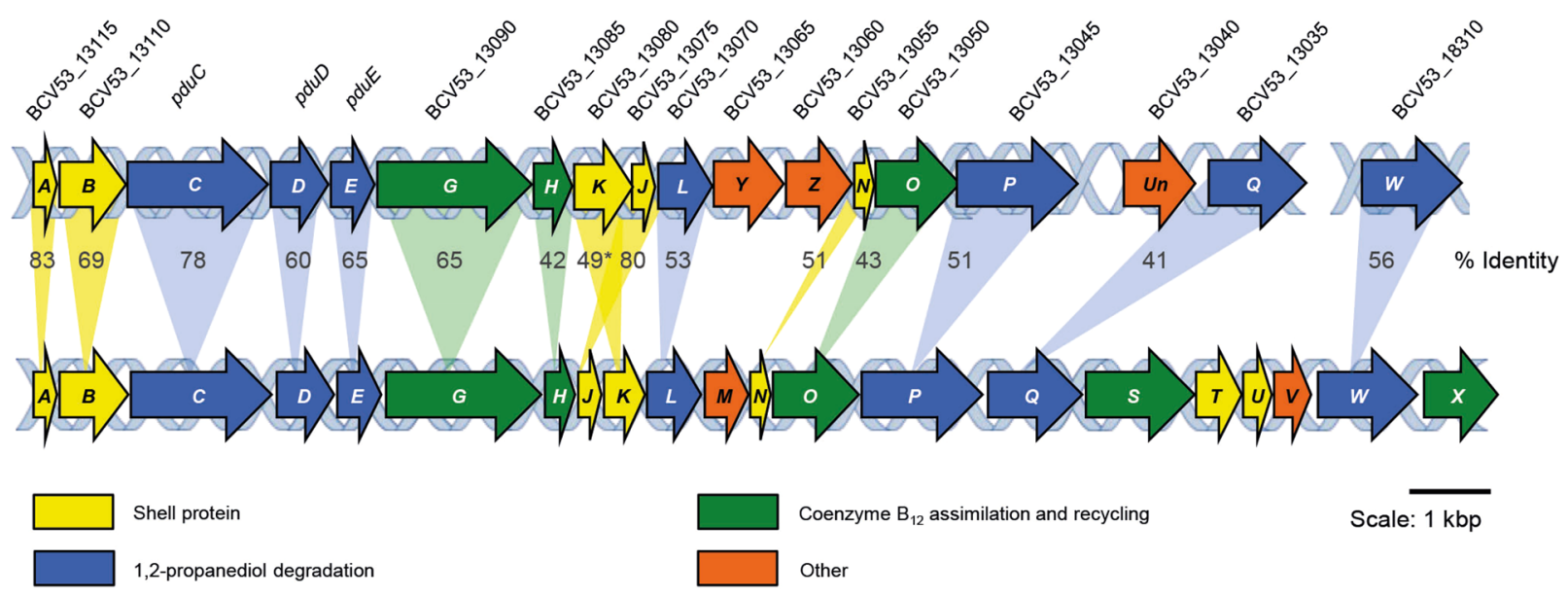

S. enterica

(b)

B. subtilis

YW01

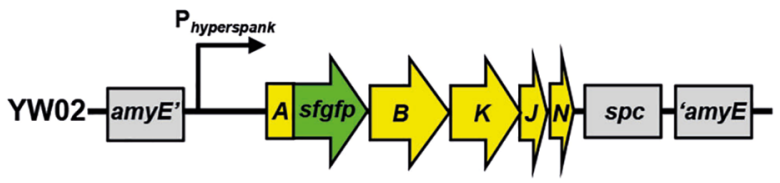

YW03

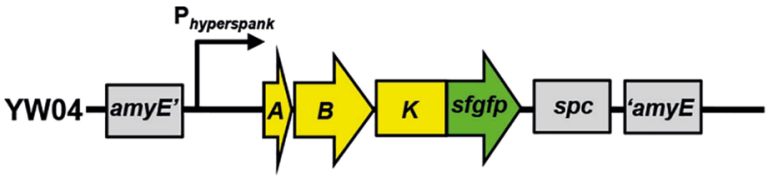

YW05

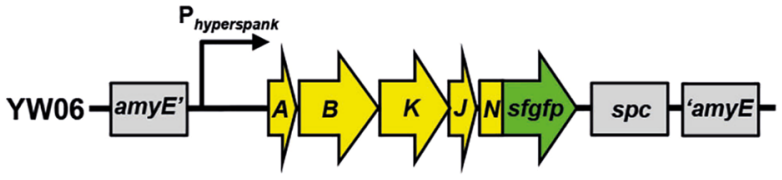

PW07-

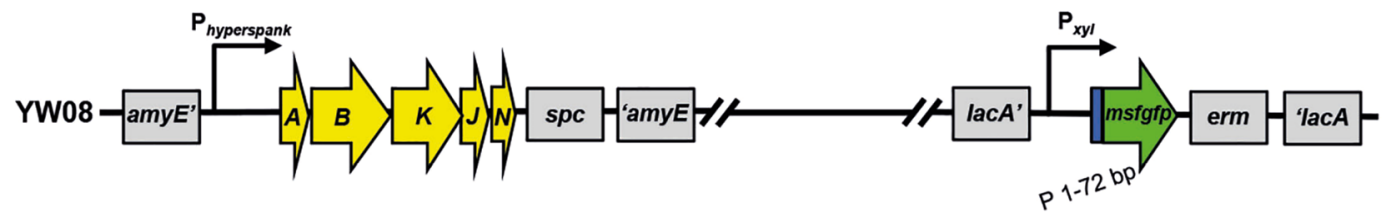

YW09-amyE'

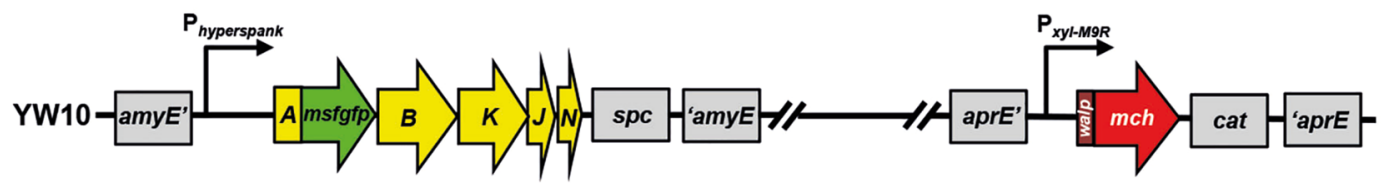

Figure 2. continued 
(c)

\section{P. thermoglucosidasius}

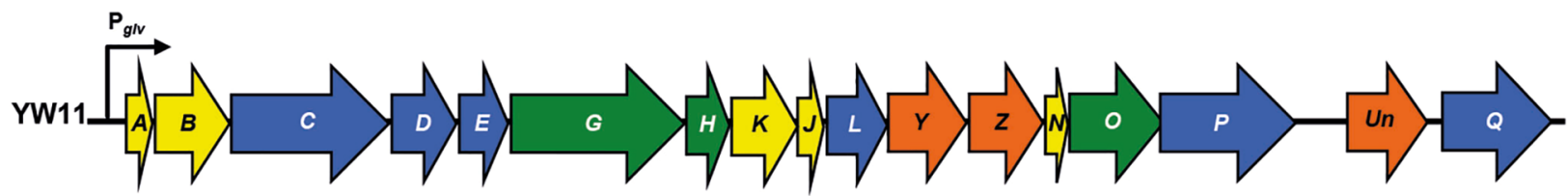

(d)

10

20

30

40

50

$P$. thermoglucosidasius (ANZ30940) SVDAQKIEKLVRKILEEMEEKK-------KPAETECEWGIFDHMNQAIE

L. innocua (WP_045553374) --ESLELEQLVKKVLLEKLAEQKEVPT--KTTTQGAKSGVFDTVDEAVQ

C. freundii (CAM57296) --NTSELETLIRNILSEQLAP----A-----KAEVKGNGIFPSVSEAID

S. enterica (AAD39015) --NTSELETLIRTILSEQLTT----PA--QTPVQPQGKGIFQSVSEAID

$$
\text { : .: * : : :** * * :.:* : }
$$

Figure 2. (a) Genomic organization of the $p d u$ operon in P. thermoglucosidasius NCIMB 11955 and S. enterica. Gene loci as identified in the $P$. thermoglucosidasius genome are given above the genes and their names, according to their functional equivalent (\% identity for each protein are shown) identified in this study, are recorded inside the arrows. The order and distribution of genes represent those identified on the chromosome. The length is an approximate relative size of the genes. $(*)$ The predicted Pdu protein from BCV53_13080 had no significant similarity to those from Salmonella based on pairwise sequence comparison but clustered within the PduK protein clade by phylogenetic analysis (Figure S1). (b) B. subtilis strains with constructed synthetic BMC operons using components of the pdu regulon from P. thermoglucosidasius NCIMB 11955 in the chromosome. (c) P. thermoglucosidasius YW11 strain with replaced the native Ppdu promoter with the Pglv promoter in the chromosome. (d) Multiple sequence alignment of PduP from $P$. thermoglucosidasius (highlighted in red) with its homologues from bacteria having a pdu metabolosome shows the presence of a longer $\mathrm{N}$-terminal extension. Only 50 residues are shown and the first amino acid (methionine) was omitted. The GenBank NCBI accession numbers are quoted in brackets.

EutN/CcmL domain-containing protein-encoding open reading frames was conducted in the 28 sequenced genomes of Geobacillus and Parageobacillus species available from the GenBank assembly database (Table S1). ${ }^{28}$ Genes encoding the shell protein homologues were discovered in eight strains: $P$. thermoglucosidasius DSM 2542 ${ }^{\mathrm{T}}$, NCIMB 11955, TM242, C56YS93, TNO-09.020, Y4.1MC1, G. thermocatenulatus KCTC 3921, and Geobacillus sp. JS12. P. thermoglucosidasius NCIMB 11955 is an important industrial and biotechnological strain for ethanol production from biomass hydrolysate and is closely related to the type strain, DSM 2542. The genome of NCIMB 11955 possesses 11 single-nucleotide polymorphisms and 2 indels compared to DSM 2542, ${ }^{29}$ whereas the improved ethanologenic $P$. thermoglucosidasius TM242 is a genetically modified version of $P$. thermoglucosidasius NCIMB 11955 . $^{30,31}$ No significant similarity to either pfam was found in the remaining species, G. stearothermophilus 10, DSM 458, G. kaustophilus HTA426, G. thermodenitrificans NG80-2, KCTC 3902, T12, G. thermoleovorans KCTC 3570, CCB_US3_UF5, FJAT-2391, ID-1, G. subterraneus KCTC 3922, G. lituanicus N3, Geobacillus genomosp. 3 JF8, Geobacillus sp. WCH70, Geobacillus sp. Y412MC52, Geobacillus sp. Y412MC61, Geobacillus sp. C56-T3, Geobacillus sp. GHH01, Geobacillus sp. 12AMOR1, and Geobacillus sp. LC300. A recent phylogenetic analysis divided members of Geobacillus into two separate monophyletic clades and the novel Parageobacillus genus was designated. ${ }^{23}$ The genes encoding shell protein homologues were evident in specific strains belonging to both Geobacillus and Parageobacillus genera. This finding suggests that the BMC was independently acquired in two phylogenetic lineages but could have been an early acquisition in $P$. thermoglucosidasius as shell protein homologues were discovered in all examined $P$. thermoglucosidasius strains. Hence, $P$. thermoglucosidasius NCIMB 11955, being the progenitor of a few industrial strains and thus having a significant potential in applied microbiology, was selected for further analysis.

Shell Proteins Are Clustered within the pdu Operon in $P$. thermoglucosidasius. Although the presence of some genes associated with the $p d u$ operon of Parageobacillus sp. Y4.1MC1 was reported as part of the genome annotation, ${ }^{32}$ description of the entire operon was not provided. To analyze the shell proteins and surrounding region, the $P$. thermoglucosidasius NCIMB 11955 genome was selected. On the basis of the presence of the conserved pfam domains, five genes, BCV53_13115, BCV53_13110, BCV53_13080, BCV53_13075, and BCV53_13055, coding for the shell homologues, were identified ${ }^{-}$in the $P$. thermoglucosidasius genome. They were situated in three distinct loci and dispersed with the other $p d u$ genes, but evidently forming a single operon, which was predicted in silico using the Softberry and ARNold software. The operon comprises 17 genes likely to be involved in 1,2-PD and ethylene glycol degradation using a cobalamin-utilizing pathway. ${ }^{33,34}$ The Pdu metabolosome has been widely characterized from Salmonella enterica, ${ }^{6}$ and is considered as the model organism in current organelle studies. A comparison of the genetic organization of the $p d u$ operons of $P$. thermoglucosidasius and S. enterica generated by pairwise amino acid alignment of translated CDSs is shown in Figure 2a. The number, diversity, and dispersion of genes in the $P$. thermoglucosidasius NCIMB 11955 operon differ from those associated with $S$. enterica but are consistent with the large variety that constitute the $p d u$ operon in a wide-range of bacterial taxa as shown previously by genome data analysis. ${ }^{3}$

Here we focus on the description of the genes encoding the shell proteins. A detailed bioinformatic analysis of the genes within the $p d u$ operon from $P$. thermoglucosidasius is given in the Supporting Information and summarized in Figure 2a. The shell proteins of the BMC, translated from BCV53_13115, BCV53_13110, BCV53_13075, and BCV53_13055 genes are 
considered to be PduA, $-\mathrm{B},-\mathrm{J}$, and $-\mathrm{N}$, respectively (Figure 2a), based on a high degree of similarity to the equivalent proteins in $S$. enterica. The expression product of the $p d u B$ gene has been reported in the literature as either PduB or both PduB and $\mathrm{PduB}^{\prime}$ proteins. ${ }^{35}$ Two potential translation start sites on the mRNA from the single $p d u B$ gene were examined in $S$. enterica and Citrobacter freundii, ${ }^{36,39}$ indicating that the PduB and $\mathrm{PduB}^{\prime}$ share identical sequences with the exception that the latter is 37 amino acids shorter. Although the putative additional start codon for $\mathrm{PduB}^{\prime}$ was observed in the $p d u B$ gene of the $P$. thermoglucosidasius genome, further proteomic analysis will be required to confirm the production of both $\mathrm{PduB}$ and $\mathrm{PduB}^{\prime}$, and consequently, we refer here to the product of $p d u B$ as simply the PduB protein. To identify a putative function for the BCV53_13080 gene, a multiple sequence alignment to the remaining potential shell proteins, PduK, -T, and -U was performed. The local alignment subtype L-INS-I, which is able to align sequences around a single alignable domain, was selected using the BLOSUM62 scoring matrix with a default gap penalty of 1.53 . From the 13 sequences, 342 alignment sites were identified with 59 gap-free and 50 conserved sites. A phylogenetic tree was constructed using only the gap-free sites, which showed that the translated BCV53_13080 gene grouped with PduKs, and had an $80 \%$ and $49 \%$ similarity to proteins from Acetobacterium woodii and $S$. enterica, respectively (Figure S1). Therefore, the order of the $p d u J$ and $-K$ genes is reversed in the $P$. thermoglucosidasius genome; a similar arrangement of shell protein genes has been reported in other Gram-positive bacteria such as Listeria innocua ${ }^{38}$ and Lactobacillus spp. ${ }^{39}$ Additionally, the two genes overlap, with the $p d u J$ translational start site being situated 4 bp upstream of the $3^{\prime}$ end the $p d u K$ gene. The genes $p d u T U$ that typically encode the rest of the shell proteins were not found in $P$. thermoglucosidasius. However, it has been previously shown that from seven $\mathrm{Pdu}$ shell proteins in Citrobacter freundii, only five, PduABJKN, were required to build the lattice of an empty recombinant BMC in E. coli. ${ }^{40}$ The essential component for formation of native BMCs with a normal structure in Salmonella, the PduM protein, ${ }^{41}$ was also not found in P. thermoglucosidasius. Despite it being reported as a shell protein in some papers, ${ }^{42}$ PduM does not possess either of the BMC or EutN/CcmL domains; it belongs to the PDUlike protein family (pfam15953), and its function for correct BMC assembly is still uncertain, and therefore, we have not considered it as a possible shell protein in this present study. Additionally, a gene encoding the $\mathrm{PduV}$ protein, which is thought to be involved in regulating the spatial distribution of BMCs in bacterial cells during growth ${ }^{40}$ was not evident in the $P$. thermoglucosidasius genome.

Shell Proteins of the Pdu BMC from $P$. thermoglucosidasius Are Expressed in $B$. subtilis. To engineer synthetic BMCs derived from the Pdu organelle of $P$. thermoglucosidasius, the biotechnologically relevant and phylogenetically close Gram-positive model organism, Bacillus subtilis, was selected. The $p d u A B,-K J,-N$ genes, coding the shell proteins of BMC, were PCR amplified from the chromosomal DNA of $P$. thermoglucosidasius NCIMB 11955 (Figure 2a) and fused in the same order that they are present in the native $p d u$ operon with the entire intergenic regions possessing the ribosomal binding sites upstream of each gene. Initially, the generated fragment was cloned on the pDR111 vector under the control of IPTG-inducible promoter, Phyperspank. To produce a stable expression system, the $p d u$ shell operon was introduced into the B. subtilis SG81 chromosome by ectopic integration into the amyE locus through double crossover homologous recombination, to give strain $B$. subtilis YW01 (Figure 2b). B. subtilis YW01c is a control strain created by transformation with the empty pDR111 plasmid (Table S2). The YW01 strain was grown in LB medium until the optical density of the culture reached 0.5 , and the expression of the synthetic shell operon was induced using $0,0.1,0.5$, or $1 \mathrm{mM}$ IPTG and incubated for a further $2 \mathrm{~h}$ at $37^{\circ} \mathrm{C}$. However, SDS-PAGE analysis of the crude cell extract did not reveal any bands corresponding to the shell protein sizes. Neither was induction of the PduA protein evident by immunoblotting, using an antibody produced against the C-terminal region of the PduA protein (residues 79-93) from Citrobacter freundii, although this may reflect a low affinity toward a heterospecific antigen. Therefore, the feasibility of expressing the BMC shell proteins from $P$. thermoglucosidasius in B. subtilis was analyzed by the detection of msfGFP using fluorescence microscopy, and Cterminal fusions of each gene, $p d u A,-B,-K,-J$ and $-N$, to msfGFP were created (Figure 2b). After chromosomal integration, this generated $B$. subtilis strains YW02, YW03, and YW06 bearing the entire shell operon with $m s f g f p$ fused after the $p d u A,-B$ and $-N$ genes, respectively (Figure $2 b$ ). The operon was interrupted in strains YW04 due to the translational overlap of $p d u K$ and $-J$ and in and YW05 as a consequence of the relatively short, intergenic region between $p d u J$ and $-N$ genes (Figure $2 \mathrm{~b}$ ). After growth and induction of B. subtilis YW02, YW03, YW04, YW05, and YW06 as described previously, cells were analyzed by fluorescence microscopy. The msfGFP signal was observed in all cultures studied confirming the successful expression of all of the shell proteins (Figure S2), at least in their $\mathrm{C}$ terminal msfGFP-tagged form. It is possible that the presence of the msfGFP enhanced the in vivo stability of the individual polypeptides, which would explain the lack of detection of natively expressed polypeptides by SDS-PAGE, but the successful incorporation of a msfGFP cargo into the lumen of a subcellular BMC (see next section) suggests that the untagged proteins are sufficiently stable to form a BMC.

B. subtilis BMCs Can Incorporate msfGFP Tagged to 24 Amino Acids from the N-Terminal Region of the PduP Cargo Protein. It has been shown that a short targeting sequence of 18 residues from the $\mathrm{N}$-terminus of $\mathrm{PduP}$ binds to the C-terminal helix of the PduA, PduJ, and PduK shell proteins and mediates incorporation in $S$. enterica and $C$. freundii MCPs. ${ }^{13,19}$ An N-terminal extension of the PduP protein from $P$. thermoglucosidasius, separated by a linker from the core enzyme, was identified by a multiple sequence alignment of its homologues in bacteria that produce BMCs, consistent with previous observations (Figure $2 \mathrm{~d}$ ). ${ }^{43}$ However, this fragment appeared to be longer than those from $S$. enterica and C. freundii. To investigate whether this $\mathrm{N}$-terminal extension facilitates the incorporation of heterologous proteins into the empty BMC in B. subtilis and to confirm the minimal length of peptide required, $p d u P(1398 \mathrm{bp})$ incorporating 72 $\mathrm{bp}(p d u P 72)$ and $54 \mathrm{bp}(p d u P 54)$ fragments from the 5 'end of $p d u P$ were $P C R$-amplified from $P$. thermoglucosidasius genome and fused to $m s f g f p$ on the pAX01 plasmid under the control of a xylose inducible promoter, Pxyl. The constructs were integrated, through double crossover homologous recombination, into the lacA region on the B. subtilis YW01 chromosome, resulting in strains B. subtilis YW07, YW08, and YW09 (Figure $2 b)$. Additionally, the same fragments were introduced into $B$. 

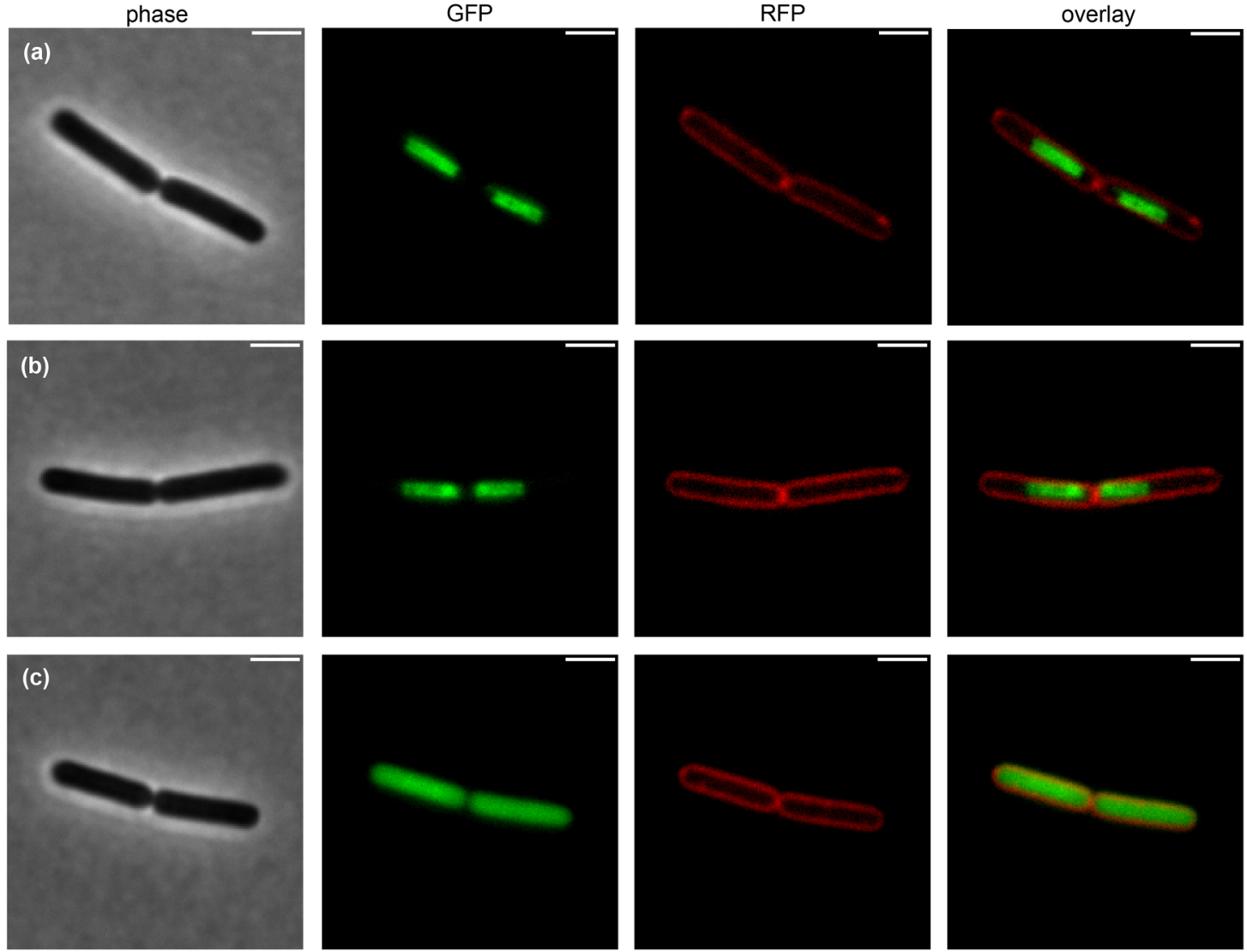

(d)
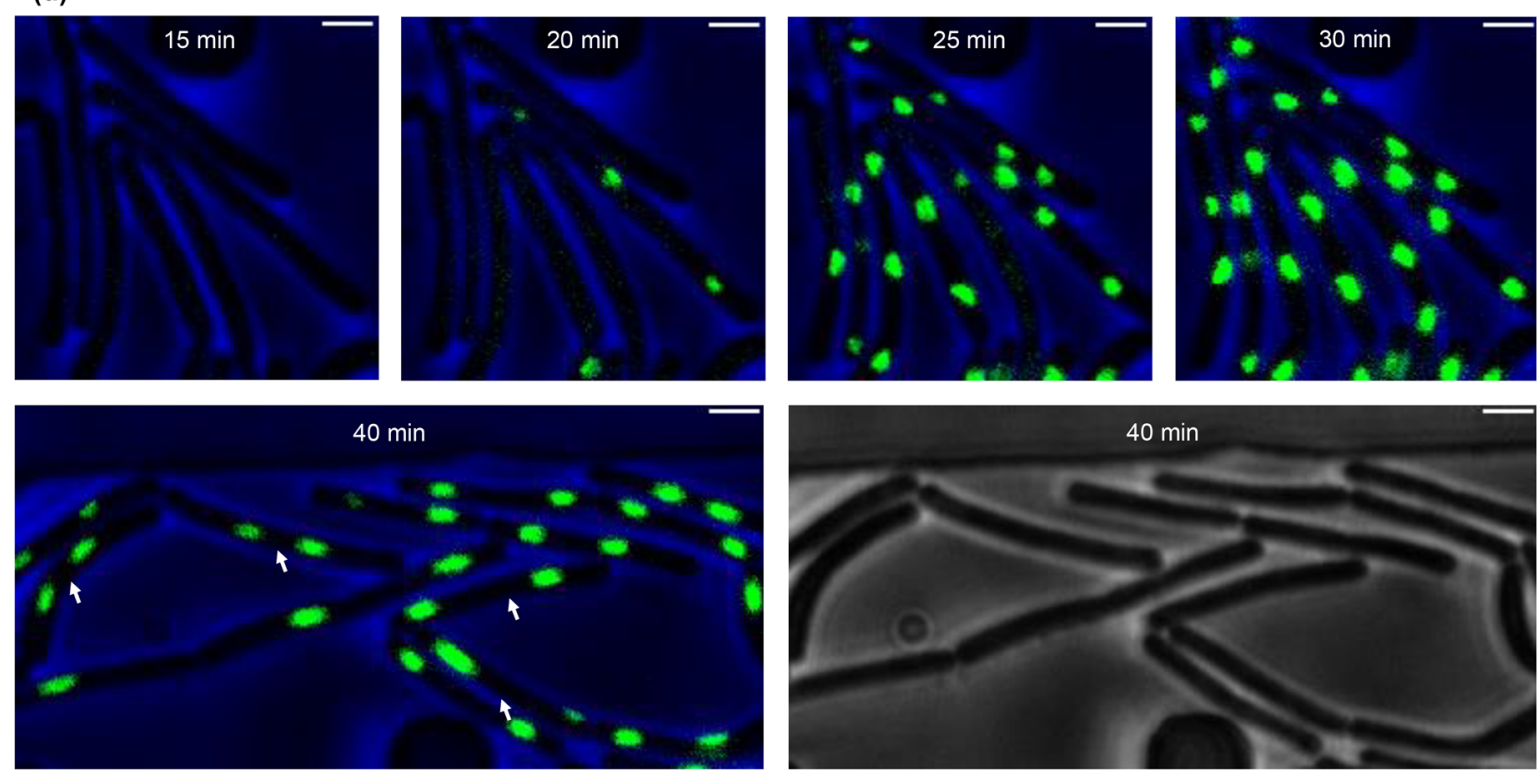

Figure 3. Fluorescent microscopy images of recombinant B. subtilis with simultaneous expression of the shell proteins and PduP-msfGFP (a), P24 msfGFP (b), and P18 msfGFP (c). The samples were visualized $1 \mathrm{~h}$ after simultaneous IPTG and xylose induction to actively growing cells. The cell membrane was visualized by staining with FM5-95 dye. The scale bar is $2 \mu \mathrm{M}$. (d) Sequential points in the synthesis of the BMCs in B. subtilis YW10 cells growing in the CellASIC B04A ONIX microfluidic chamber visualized by enhancing the contrast and using pseudocolored imaging. The composite figures display the results of the merged brightfield (blue) and GFP (green) channels. White arrows indicate the position of the septa between the cells, defined by an mCherry-labeled cell membrane. The scale bar for all pictures represents $2 \mu \mathrm{M}$.

subtilis YW01c providing the control to give B. subtilis YW07c, YW08c, and YW09c strains, respectively (Table S2). For fluorescence imaging of the B. subtilis YW07, YW08, and YW09 and control strains, bacteria were cultivated in LB medium, as the xylose inducible promoter was identified to be subject to catabolite repression and, thus, the fluorescence signal was not detectable in glucose-containing medium. Coproduction of the shell proteins and msfGFP fusion proteins with 24 (P24-) and 18 (P18-) N-terminal amino acid extensions derived from $\mathrm{PduP}$ and full length $\mathrm{PduP}$ 


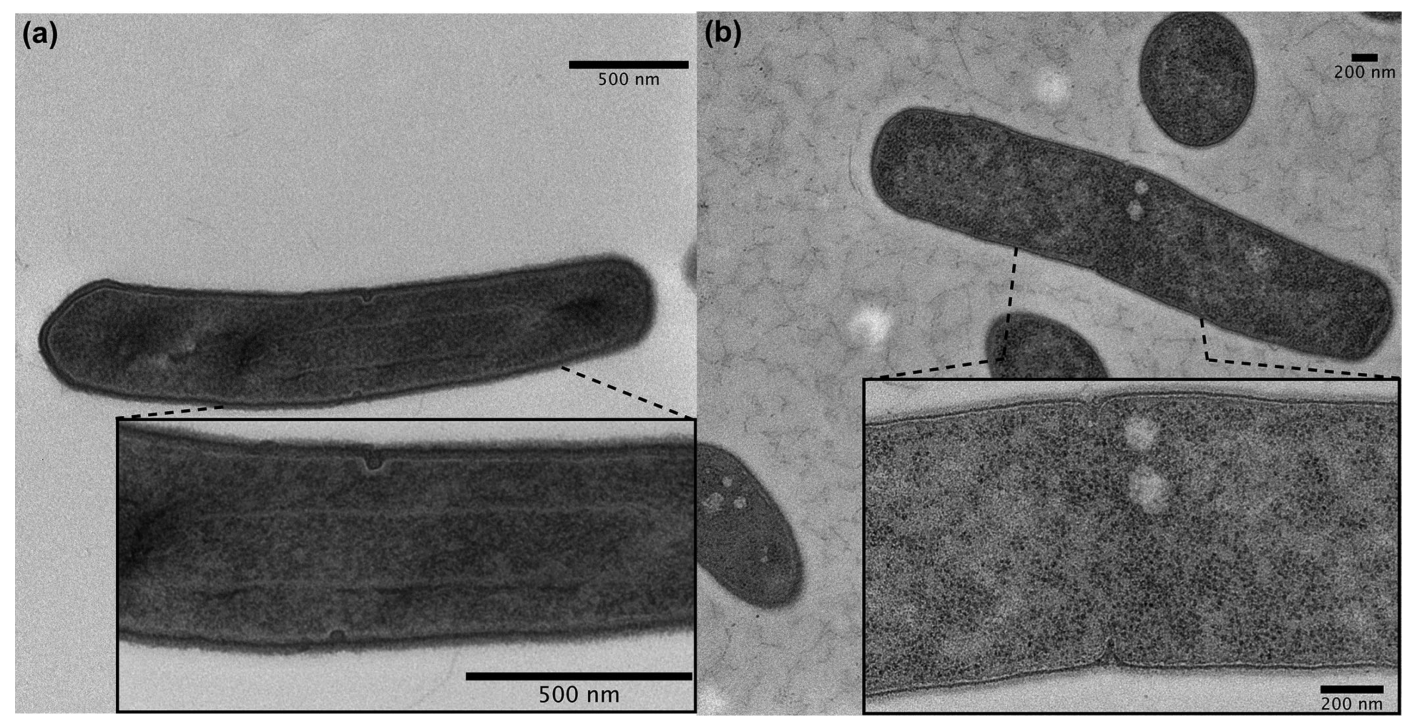

Figure 4. TEM longitudinal ultrathin sectioning visualizes the entire large centrally localized BMC in B. subtilis YW01 (a) and the low dense polyhedral organelles in P. thermoglucosidasius YW11 (b) cells. The cultures were grown in LB medium and processed for analysis after $1 \mathrm{~h}$ upon the synthetic shell and the native $p d u$ but with switched promoter region operons, respectively.

(PduP-) was carried out by the addition of both IPTG and xylose. The simultaneous expression of proteins from the shell operon and both PduP- or P24- proteins fused to msfGFP resulted in the development of a localized fluorescent signal associated with tube-like structures observed under fluorescence microscopy, indicating successful BMC formation (Figure 3a,b). In contrast, the coexpression of the shell and P18 msfGFP fusion proteins showed the signal was evenly distributed across the entire cell, indicating the loss of targeting to the BMC (Figure 3c). The appearance of the same msfGFP signal distributed across the cytosol was observed in $\mathrm{YW07c}$, YW08c, and YW09c cells in which the BMC shell operon was absent.

Although the P24 or whole length PduP-msfGFP fusions clearly showed protein localization in a compartment formed by expression of the $P$. thermoglucosidasius BMC shell proteins, the morphology of these BMCs was different from those that have been previously reported. A single BMC was observed in each cell and in $84 \%$ cells in total $(n=131)$. The organelles were tubular in appearance and the mean length of the 110 measured BMCs was $3.18 \mu \mathrm{m}$ (s.d. $1.14 \mu \mathrm{m}$ ) with a range of $4.76 \mu \mathrm{m}$. The BMCs were mostly positioned centrally within the cells, appearing shorter when at the division poles (minima: $0.76 \mu \mathrm{m}$ ) before enlargement after moving to the center of the cell during bacterial growth (maxima: $5.52 \mu \mathrm{m}$ ). Binning the data $(n=110$, bins $=6)$ indicates that the largest proportion (29\%) of lengths clustered around the data median of $3.01 \mu \mathrm{m}$.

Assembly of BMCs Occurs after 20 min of Shell Operon Induction. To establish the time required to build an empty shell BMC in B. subtilis cells, time-lapse fluorescence microscopy was conducted. To label the membranes of $B$. subtilis and identify the cell outlines in vivo, ${ }^{44}$ an artificial transmembrane domain containing 23 amino acids, WALP, ${ }^{45}$ was fused to mCherry and integrated into the aprE locus in YW02, resulting in strain YW10. The sequence of induction of msfGFP-labeled BMCs for B. subtilis YW10 grown in MCGT medium at $32{ }^{\circ} \mathrm{C}$ in a microfluidic plate is shown in Figure $3 \mathrm{~d}$ and Video S1. After an adaptation period to allow growth and division under experimental conditions, the IPTG containing medium was moved to an incubation chamber. The msfGFP signal was observed in the bacterial cytoplasm 15 min after shell operon induction. A few defined fluorescent puncta were observed at $20 \mathrm{~min}$, and after $35 \mathrm{~min}$ of incubation the signal became completely localized and was present in the majority of visualized cells, indicating BMC assembly.

Transmission Electron Microscopy (TEM) Analysis Confirms the Presence of Large Tubular BMCs in $B$. subtilis Cells. The analysis of msfGFP targeted to coinduced BMCs suggested that the BMC was forming a single tubular compartment (Figure 3a,b). To obtain direct evidence for this BMC structure in the absence of a cargo protein, cells were sectioned and examined by TEM. Long tubular structures were observed by TEM analysis of ultrathin sections of B. subtilis YW01 strain after induction of the shell operon (Figure 4a, Figure $\mathrm{S} 3 \mathrm{a}-\mathrm{c}$ ) and were comparable to those seen by light microscopy. Only a few full length BMCs were observed due to the small number of longitudinal cells obtained from embedding in a random orientation and thin sectioning sample preparation. These had an average length of $1.4 \mu \mathrm{m}$. Examination of a similar number of cells in the control YW01c strain (empty vector) did not reveal any intracellular structures. The BMC localization pattern was similar to that in experiments using fluorescence microscopy in which cells had a single BMC localized toward the center of the cells. In cross-sectional orientations the compartments were ellipsoidal with an average maximal $270 \mathrm{~nm}(n=23, \mathrm{SD}=70)$ and minimal $180 \mathrm{~nm}(n=23, \mathrm{SD}=40)$ diameter.

BMCs Are Produced in $P$. Thermoglucosidasius at $\mathbf{6 0}$ ${ }^{\circ} \mathrm{C}$. While the $P$ thermoglucosidasius shell proteins clearly formed compartments in B. subtilis, their appearance was different from those currently observed in native or recombinantly produced BMCs in mesophilic organisms. This might have been as a result of either the different expression levels of the shell proteins in B. subtilis, compared to the native host or due to the thermophilic nature of heterologous proteins. Therefore, it was of interest to compare the shape, size, and intracellular localization of the empty $\mathrm{BMCs}$ to those in its natural thermophilic host at $60{ }^{\circ} \mathrm{C}$. Owing to the complications of obtaining reliable induction of a 
catabolite-repressible operon in $P$. thermoglucosidasius, the native $\mathrm{P} p d u$ promoter was replaced with a $\mathrm{Pglv}$ maltose inducible promoter, producing the strain $P$. thermoglucosidasius YW11 (Figure 2c). This was cultivated aerobically at its optimal growth temperature of $60{ }^{\circ} \mathrm{C}$ to the mid-exponential growth phase, whereupon the $p d u$ regulon was induced by the addition of $1 \%$ maltose, and samples were collected for TEM after $1 \mathrm{~h}$ of induction. The parent strain $P$. thermoglucosidasius NCIMB 11955 cultivated under the same conditions was also processed. The development of clearly defined polyhedral intracellular structures in the ultrathin-sectioned YW11 strain was evident, in contrast to the wild type strain in which those were absent (Figure 4b, Figure S3d-f). Between one and nine BMCs per cell were observed. These organelles had a similar morphology in both transverse and longitudinal views. The mean diameter of the metabolosomes measured was $125 \mathrm{~nm}$ across their widest point $(n=46, \mathrm{SD}=49 \mathrm{~nm})$. Considering the ratio of maximum to minimum diameters, two clusters were observed with one group $(n=36)$ having shorter nearspherical structures $(77-145 \mathrm{~nm})$ and a smaller group $(n=$ 10) of irregular polyhedrals with longer (165-297 nm) maximum and shorter $(105-257 \mathrm{~nm})$ minimum diameters. It appears that as compartments increase in size their shape becomes less spherical, as evidenced by the increasing ratio between the maximum and minimum diameters. These observations correlate with previous studies that show stark differences between the dimensions of wild-type metabolosomes in S. enterica as well as recombinant compartments derived from $C$. freundii in E. coli cells broadly fitting into two size groups. ${ }^{36,37}$ The BMCs appeared to be randomly distributed in the cytoplasm without any defined cell localization.

\section{DISCUSSION}

This study has identified genes encoding homologues of BMC shell proteins in thermophilic representatives of Bacillaceae, Geobacillus, and Parageobacillus spp. The structure of the $p d u$ operon from $P$. thermoglucosidasius is similar to those previously described from Gram-positive microorganisms but differs from $S$. enterica. It includes two new genes, given the identifiers, $p d u Y$ and $p d u Z$, which encode proteins of unknown function that are probably associated with the operation of BMCs in thermophiles while a third new gene, $p d u U n$, encodes a protein of unknown function that is uniquely associated with members of the Bacillaceae family.

On the basis of previous heterologous expression studies with BMCs from $S$ enterica, it was shown that expression of the Pdu shell proteins, PduABKJN in B. subtilis produced large, empty proteinaceous organelles. These differ from the polyhedral bodies observed in P. thermoglucosidasius but it is known that altering the expression of shell proteins can produce different subcellular architectures that could potentially be customized to have explicit functions. Despite a large amount of research, ${ }^{11,12,16}$ the factors controlling assembly of protein subunits into BMCs of different sizes and shapes are still poorly understood. It has been recognized that BMC morphology is dependent on the ratio of the shell proteins from which they are formed. So, heterologous overexpression of PduA, either together with the rest of shell proteins or in their absence, led to the development of tube-like structures in E. coli cells. ${ }^{40,46}$ Similarly, cell length tubes were produced in $E$. coli by the heterologous expression of a single shell protein derived from the naturally circular BMCs of unknown function from Mycobacterium smegmatis. ${ }^{47}$ The recently determined crystal structure of a BMC shell suggests that hexamers and pseudohexamers form different subunit-subunit angles, and thus the size of the compartment depends on the various combinations of distinct BMC domains. Although shells with different sizes corresponding to the range of size to the natural BMC (40-600 nm) could be modeled using these protein arrangements, $^{12}$ it is unclear how these construction principles extend to BMCs that lack icosahedral architecture. Additionally, the size of the BMC shells is often inconsistent with their cargo, in that empty shells have been found to be larger or smaller than their fully loaded shells in different wild type and recombinant systems. ${ }^{48,49}$ A recent atomic-resolution model of an intact BMC shell composed of five different proteins from Haliangium ochraceum with an inner diameter of $290 \AA$, estimated that the volume of a BMC lumen could include about 150 copies of a $60-\mathrm{kDa}$ enzyme. ${ }^{12}$ Therefore, larger recombinant BMCs could provide the opportunity to load the organelle with multienzymatic pathways, enhancing their metabolic efficiency.

Previous studies have shown that 18 residues on the $\mathrm{N}$ terminus of the PduP and PduD, and $\mathrm{N}$-terminal extensions of 20 amino acids of the PduL, directed fluorescent proteins into the lumen of $S$. enterica or $C$ freundii BMCs. ${ }^{25,43,50}$ Furthermore, fusion of the PduP18 and PduD18 encapsulation peptides to pyruvate decarboxylase and alcohol dehydrogenase resulted in the development of compartments as ethanolproducing bioreactors, which enhanced alcohol yield compared to the untagged cytoplasmic enzymes, demonstrating the advantage of encapsulation of the process within BMC. ${ }^{19}$ This would be interesting to investigate with thermostable pyruvate decarboxylases (Pdc) as it has proven difficult to express a thermophilic Pdc-dependent ethanol pathway at high temperatures in $P$. thermoglucosidasius. ${ }^{51-53}$

It has been reported that conserved hydrophobic motifs from several different systems, such as the ethanolamine utilization and glycyl radical-generating BMCs, encapsulate heterologous proteins within the PduP compartment in $S$. enterica. ${ }^{18}$ Another study, in Corynebacterium glutamicum, has shown that it is possible to target reporter proteins, fused to synthetic scaffold interaction partners (PDZ and GTPase protein binding domain), using a C-terminal tag, which could be advantageous for the construction of BMCs, when $\mathrm{N}$ terminal targeting is not possible. ${ }^{54}$ Recently, a new approach using de novo coiled-coil protein-protein interactions has been developed. The PduA protein was redesigned such that the $\mathrm{N}$ terminal sequence was redirected on the luminal side of the $\mathrm{BMC}$, without influencing the formation of the BMC. Recombinant proteins were then tagged into the lumen of the BMC through the designed coiled-coil system. ${ }^{55}$

With our expanding knowledge of approaches to target recombinant proteins to the lumen of BMCs, it should be possible to load the empty organelles developed in this work with multienzyme pathways and, subsequently, to utilize them as intracellular bioreactors within B. subtilis. Temperature effects on assembly might alter configuration, but this is yet to be tested. Assembly in B. subtilis offers the opportunity to generate novel BMC-enzyme combinations in a genetically tractable system. These can then be transferred to $P$. thermoglucosidasius if desired. Synthetic biology approaches using a narrow group of proteins across a range of different organisms provide the opportunity to develop many promising applications in industrial and medical biotechnology. ${ }^{56-58}$ 
Much effort has been made to engineer BMCs into nanoreactors to enhance product turnover by increasing metabolic pathway flux and isolate toxic reaction intermediates from the rest of the cell. ${ }^{59,60}$ Coupled with the prospect of applying compartmentalization to deliver a commercial standard of products such as vaccines, advance biofuels, and fine chemicals, the development of larger BMCs presents a significant and realistic opportunity for industry. ${ }^{61-63}$

\section{METHODS}

Genomic Data Analysis. To search for shell protein homologues, the seed alignment of the BMC pfam00936 (429 sequences) and EutN/CcmL pfam03319 (194 sequences) domain families was downloaded from the Pfam database. ${ }^{64}$ The shell protein homologues in the genomes of Parageobacillus and Geobacillus spp. were searched using the BLASTP (v2.8.0+) online tool ${ }^{65}$ against the Nonredundant Protein Database, comprising GenBank Coding Sequence (CDS) translations, Protein DataBank, SwissProt, Protein Information Resource, and Programmed Ribosomal Frameshift, excluding environmental samples from Whole Genome Sequence projects, February 1, 2019. All hits with an E-value less than $1 \times 10^{-12}$ and percentage identity of $50 \%$ or greater were accepted as potential BMC shell protein homologues. Identical proteins matching genomes associated with these hits were analyzed, and only results for bacteria with completely assembled genomes were selected. The genomic locus, comprising 20 genes to the left and to the right of $p d u N$, was selected, and the putative operon was predicted by finding promoter and transcriptional terminators using the Softberry BPROM (http://www.softberry.com/) and ARNold ${ }^{66}$ online tools, respectively. Multiple protein sequence alignments were generated using MAFFT (v7) ${ }^{67}$ online multiple sequence alignment tools. The neighbor-joining method and JTT substitution model ${ }^{68}$ was used to construct a phylogenetic tree, with a resampling number of 100 using the bootstrap technique. The constructed tree was exported as a phyloXML object and annotated using the Interactive Tree of Life (IToL v3) ${ }^{69}$ web-based software. The potential homologues of the translated open reading frames of the $p d u$ operon from $P$. thermoglucosidasius NCIMB 11955 (NCBI RefSeq sequence: NZ_CP016622) were searched against Salmonella spp. (NCBI taxonomic identifier: 590) using the aforementioned BLAST protocol (unless otherwise stated in the text).

General Methods and Strain Construction. The descriptions of the bacterial strains used in this study are provided in Table S2. E. coli and B. subtilis were routinely maintained on nutrient agar (Oxoid) solid medium and Lysogeny broth (LB) at $37^{\circ} \mathrm{C}$. The media, where necessary, were supplemented with the following antibiotics $\left(\mu \mathrm{g} \mathrm{mL}^{-1}\right)$ : ampicillin, 100; kanamycin, 25; spectinomycin, 60; erythromycin, 1. $P$. thermoglucosidasius was grown in tryptic soy agar (TSA) and liquid tryptic soy broth or $2 \times$ Yeast Tryptone media at $60{ }^{\circ} \mathrm{C}$. Chemicals and reagents were purchased from Sigma-Aldrich. Bacterial genomic DNA was extracted from 3 $\mathrm{mL}$ of culture at the late exponential phase of growth using Wizard Genomic DNA purification kit (Promega). Plasmid DNA was isolated from $4 \mathrm{~mL}$ of culture using QIAprep Spin Miniprep kit (Qiagen) according to the protocol provided. Polymerase chain reaction (PCR) products were purified using the QIAquick PCR Purification Kit (Qiagen). The relevant restriction enzymes and T4 DNA ligase were purchased from NEB. The plasmids and primers used in this work are listed in
Tables S3 and S4, respectively. The genes, $p d u A B,-K J$ and $-N$, coding shell proteins were amplified from $P$. thermoglucosidasius NCIMB 11955 genomic DNA using the 01/02, 03/04, and $05 / 06$ primer pairs, and fused by overlapping $\mathrm{PCR}^{70}$ to create an artificial operon. Q5 High-Fidelity DNA Polymerase (NEB) was used for PCR with reaction conditions according to the manufacturer's protocol. The specific recognition nucleotides for BmtI and SphI restriction enzymes were introduced into the primer sequences of 01 and 06 , respectively. The resulting fragment was cloned into the $B m t \mathrm{I}$ and $S p h \mathrm{I}$ restriction sites on vector $\mathrm{pDR} 111$. MsfGFP was introduced by the MEGAWHOP technique $^{71}$ using The KOD Xtreme Hot Start DNA Polymerase (Merck Millipore). The $p d u P$ gene and its truncated versions comprising 72 and 54 base pairs were amplified from the $P$. thermoglucosidasius NCIMB 11955 chromosome using $17 / 18,17 / 19$, and $17 / 20$ primers, respectively. The $P$ shAI and SphI restriction sites were introduced into 17 and 18, 19, 20 primers, respectively, and the fragments were cloned into the corresponding sites on the pYW07 vector. For replacement of the native $p d u$ by the $g l v$ promoter, the $g l v$ promoter region of $P$. thermoglucosidasius was amplified from the P2 plasmid using 21/22 primers. The regions surrounding the native $p d u$ promoter, BCV53 13120 and $p d u A$, needed for homologous recombination were amplified by $23 / 24$ and $25 / 26$ primers, respectively. The resulting products were cloned into pG2KoriT by Golden Gate Assembly (NEB). E. coli DH5 $\alpha$ strain was used for vector construction and transformation, which were carried out using the standard protocol. ${ }^{72}$ B. subtilis cell competence and transformation were conducted as described by Hamoen and coauthors. $^{73}$ B. subtilis SG81 was created by Daniel et al., differing from the $B$. subtilis 168 organism by the insertion of a neo gene in lacA within its chromosome, which was subsequently used for strain construction in this study. ${ }^{74}$ Transformants with double-crossover integration into the $a m y E$ region of $B$. subtilis were selected for their acquired resistance to spectinomycin and loss production of $\alpha$-amylase and so their inability to form clear zones by cultivation on nutrient agar plates supplemented with $0.05 \%$ of soluble starch. Recombination into the lacA site was confirmed by replacement of the kanamycin resistance by erythromycin and PCR using $27 / 28$ primers. $P$. thermoglucosidasius was transformed by conjugation using the E. coli S17-1 $\lambda$ pir strain bearing the pYW11 vector. For this, $P$. thermoglucosidasius and E. coli cultures, freshly grown to an optical density of 0.5 , were mixed in a 9 to 1 ratio and directly transferred onto TSA medium and cultivated at $37{ }^{\circ} \mathrm{C}$ for $16 \mathrm{~h}$. The exconjugants were selected following incubation on TSA plates supplemented with kanamycin $(12 \mu \mathrm{g} / \mathrm{mL})$ at $52{ }^{\circ} \mathrm{C}$.

Fluorescence Microscopy. Bacteria were grown in SMMdefined minimal medium supplemented with $0.4 \%$ casamino acids, $1 \%$ glucose, and $2 \%$ tryptophan (MCGT) (unless otherwise stated) at $37{ }^{\circ} \mathrm{C}$. Gene expression was induced by the addition of $1 \mathrm{mM}$ isopropyl- $\beta$-D-thiogalactopyranoside (IPTG) and/or $1 \%$ xylose when the optical density of the culture reached 0.5 . A bacterial culture $(2 \mu \mathrm{L})$ was mounted on a slide covered with a thin layer of $1.2 \%$ agarose in deionized water. Cell membranes were stained with $0.5 \mu \mathrm{g} / \mathrm{mL}$ FM5-95 dye (Invitrogen). Visualization of the samples was carried out using a Nikon Eclipse Ti microscope equipped with a PlanApochromat objective (Nikon DM 100x/1.40 Oil Ph3). Light was transmitted from a $300 \mathrm{~W}$ xenon arc-lamp through a liquid light guide (Sutter Instruments), and images were collected 
using a CoolSnap HQ2 cooled CCD camera (Photometrics). METAMORPH imaging software (version V.6.2r6) was used to capture and analyze the digital images.

Fluorescence Time-Lapse Imaging. Time-lapse fluorescence microscopy, using the CellASIC B04A ONIX Microfluidic Plates (Merck Millipore), was performed according to the protocol of Santos-Beneit et al. ${ }^{75}$ with the following modifications: bacteria were cultivated in MCGT medium at $30^{\circ} \mathrm{C}$ overnight. The following morning the culture was diluted with fresh medium to $\mathrm{OD}_{600} 0.05$ then incubated further until the optical density reached 0.3 ; cells were then loaded into the microfluidic growth chamber at 4 psi for $15 \mathrm{~s}$, followed by a wash for $30 \mathrm{~s}$ at 3 psi. The plate was placed into a heated microscope chamber maintained at $32{ }^{\circ} \mathrm{C}$. Continuous flow of media through the device was maintained at 2 psi throughout the experiment. The media containing $1 \mathrm{mM}$ IPTG was used for the shell operon induction. The samples were visualized using a Nikon $\mathrm{Ti}$ microscope fitted with a Nikon Plan Apo objective (100x/1.40 Oil Ph3). The illumination settings were GFP $150 \mathrm{~ms}$ and mCherry 200 ms. The images were autoacquired at $5 \mathrm{~min}$ intervals using FRAP-AI ${ }^{76}$ and processed with ImageJ (v1.52a) software. ${ }^{77}$

Thin Sections of Cells and Transmission Electron Microscopy (TEM). P. thermoglucosidasius and B. subtilis strains for TEM microscopy were plated on nutritient agar and incubated overnight at 60 and $37{ }^{\circ} \mathrm{C}$, respectively. The following morning liquid cultures of bacteria were set up in 25 $\mathrm{mL}$ of $\mathrm{LB}$ in $125 \mathrm{~mL}$ baffled flasks and cultivated at the aforementioned temperatures, with shaking at $250 \mathrm{rpm}$ and further diluted in fresh medium to equal a cell optical density of 0.05 . When the $\mathrm{OD}_{600}$ of $P$. thermoglucosidasius $\mathrm{BMC}$ and $B$. subtilis BMC01 cultures reached $0.4,1 \%$ maltose or $1 \mathrm{mM}$ IPTG was added into the cultivation medium, respectively. The samples for TEM were collected from both cultures after 1 h of induction. P. thermoglucosidasius NCIMB 11955 and B. subtilis SG81 cultivated under the same conditions were also processed. Strains for thin sectioning were processed using the same protocol but with an extra step for P. thermoglucosidasius, where cells were encapsulated in 3\% agar. Ten milliliter cultures were pelleted by centrifugation at $4000 \mathrm{~g}$ for $5 \mathrm{~min}$ at room temperature and washed twice with $2.5 \%$ glutaraldehyde in $0.1 \mathrm{M}$ sodium cacodylate buffer $(\mathrm{pH} 7.2)$. The pellet was then resuspended in $1 \mathrm{~mL}$ of the same buffer and fixed overnight at $4{ }^{\circ} \mathrm{C}$. Bacteria were incubated in $1 \%$ osmium tetroxide in sodium cacodylate buffer for $1 \mathrm{~h}$ with a subsequent wash in $\mathrm{dH}_{2} \mathrm{O}$ three times for $20 \mathrm{~min}$. Samples were dehydrated in a series of acetone concentrations as follows: $50,70,80,90$, and $100 \%$ acetone three times for $15 \mathrm{~min}$. Samples were infiltrated in a 1:2 spurr resin and dry acetone for $1 \mathrm{~h}$ with two changes, 1:1 spurr resin and dry acetone overnight, $100 \%$ spurr resin with vacuum treatment for $8 \mathrm{~h}$, and $100 \%$ spurr resin overnight. Cells were embedded into spurr resin in molds and polymerized at $70{ }^{\circ} \mathrm{C}$ for $8 \mathrm{~h}$. Bacterial blocks were thin sectioned on a Leica EM UC7 ultramicrotome and machine stained with $1 \%$ uranyl acetate and lead citrate. Sections were examined in the Philips CM 100 Compustage (FEI) transmission electron microscope at an accelerating voltage of $100 \mathrm{kV}$. Digital images were collected using an AMT CCD camera (Deben).

SDS-PAGE and Western Blot. Fresh bacterial cultures, cultivated in LB medium either supplemented with IPTG or in the absence of an inducer for $2 \mathrm{~h}$, were harvested by centrifugation at $6000 \mathrm{~g}$ for $3 \mathrm{~min}$. The pellet was resuspended in $50 \mathrm{mM}$ Tris- $\mathrm{HCl}, \mathrm{pH} 8.0$ buffer supplemented with $0.6 \mathrm{M}$ sucrose, and $5 \mathrm{mM}$ EDTA and incubated with lysozyme (2 $\mathrm{mg} / \mathrm{mL}$ ) at $37{ }^{\circ} \mathrm{C}$ for $2 \mathrm{~h}$. Protoplasts were pelleted by centrifugation at $7700 \mathrm{~g}$ for $15 \mathrm{~min}$ and resuspended in sonication buffer (50 mM Tris-HCl, pH 7.9, 2 mM EDTA, 150 $\mathrm{mM} \mathrm{NaCl}$, Complete Protease Inhibitor Cocktail (Roche)) and sonicated three times $(10 \mathrm{~s} \mathrm{ON}$, followed by a $1 \mathrm{~min}$ interval). Laemmli buffer $(4 \times)$ and 10X Reducing Agent were added and samples were incubated at $80^{\circ} \mathrm{C}$ for 5 min. Proteins were separated by sodium dodecyl sulfate polyacrylamide gel electrophoresis (SDS-PAGE) using a NuPAGE Bis-Tris gel with polyacrylamide percentages from 4 to $12 \%$ in $1 \times$ MES Running Buffer (ThermoFisher Scientific) in Novex midi cells (Invitrogen). Protein bands were visualized using Instant Blue protein stain (Expedeon, Cambridge, UK).

Electroblotting to a Hybond-P PVDF membrane (GE Healthcare) was performed using a semidry apparatus (Hoefer Scientific Instruments) at a constant $75 \mathrm{~V}$ for $60 \mathrm{~min}$. The blocking was performed by incubation with $2 \%$ BSA in PBST (10 mM Na $2 \mathrm{HPO}_{4}, 1.8 \mathrm{mM} \mathrm{KH} \mathrm{PO}_{4}, 137 \mathrm{mM} \mathrm{NaCl}, 0.05 \%$ Tween-20, pH 7.4) buffer at $4{ }^{\circ} \mathrm{C}$ for $12 \mathrm{~h}$. The membrane was incubated with either anti-PduA rabbit IgG (1:500) or PBP1 (1:1000) primary antibody in PBST with $0.4 \%$ BSA with continuous agitation at $25{ }^{\circ} \mathrm{C}$ for $2 \mathrm{~h}$. The membrane was washed twice for $5 \mathrm{~min}$ with PBST, and the goat antirabbit horseradish peroxidase-conjugated secondary antibody (1:10000) was incubated for another $60 \mathrm{~min}$, washed three times for $5 \mathrm{~min}$ and the color was developed using the Pierce ECL Plus Substrate (Thermo Scientific). Samples were visualized by an ImageQuant LAS 4000 mini digital imaging system (GE Healthcare).

\section{ASSOCIATED CONTENT}

\section{Supporting Information}

The Supporting Information is available free of charge on the ACS Publications website at DOI: 10.1021/acssynbio.9b00155.

Figures S1-S3; Tables S1-S4; bioinformatic analysis; references $(\mathrm{PDF})$

Video S1 (AVI)

\section{AUTHOR INFORMATION}

\section{Corresponding Author}

*E-mail: wade.yana@gmail.com.

ORCID

Yana Wade: 0000-0003-1950-149X

David J. Leak: 0000-0003-1578-1041

\section{Author Contributions}

This study was funded through a Marie Curie Individual Fellowship awared to YW, which was initially conceived by YW and DJL. RAD and YW designed the research programme performed during a secondment of YW to Newcastle. YW performed the bioinformatic analysis, designed and conducted the experiments, and interpreted the results on both Parageobacillus and Bacillus with guidance from DJL and RAD, respectively. YW wrote the manuscript with input from all.

\section{Notes}

The authors declare no competing financial interest. 


\section{ACKNOWLEDGMENTS}

This research was supported by a Marie Skłodowska-Curie Individual Fellowship (Call: H2020-MSCA-IF-2015, 704724) granted to $\mathrm{YW}$; RAD acknowledges the support of the EPSRC Programme Grant (EP/N031962/1); DJL acknowledges support from the EPSRC (EP/K014889/1) and BBSRC (BB/J001120/2) Programme Grants. We thank Dr. Matthew Wade (Newcastle University) for bioinformatic support. We thank Prof. Jeffery Errington, Dr. Henrik Strahl (Newcastle University), and Prof. David Rudner (Harvard Medical School) for supply of the plasmids used in this work and are grateful to Prof. Martin Warren (University of Kent) for the anti-PduA antibody gift. We also thank Ursula Potter and Diana Lednitzky at the Microscopy and Analysis Suite, University of Bath, for help in preparation of the $P$. thermoglucosidasius samples for TEM; Tracey Davey and Ross Laws at the Electron Microscopy Research Services, Newcastle University for processing of B. subtilis cells, and conducting thin sectioning and TEM analysis of samples; Isabel Huston for help in undertaking the experimental work on promoter exchange in $P$. thermoglucosidasius.

\section{REFERENCES}

(1) Bobik, T. A., Lehman, B. P., and Yeates, T. O. (2015) Bacterial microcompartments: widespread prokaryotic organelles for isolation and optimization of metabolic pathways. Mol. Microbiol. 98, 193-207.

(2) Craciun, S., and Balskus, E. P. (2012) Microbial conversion of choline to trimethylamine requires a glycyl radical enzyme. Proc. Natl. Acad. Sci. U. S. A. 109, 21307-21312.

(3) Axen, S. D., Erbilgin, O., and Kerfeld, C. A. (2014) A taxonomy of bacterial microcompartment loci constructed by a novel scoring method. PLoS Comput. Biol. 10, No. e1003898.

(4) Cannon, G. C., Heinhorst, S., and Kerfeld, C. A. (2010) Carboxysomal carbonic anhydrases: Structure and role in microbial CO2 fixation. Biochim. Biophys. Acta, Proteins Proteomics 1804, 382392.

(5) Shively, J. M., English, R. S., Baker, S. H., and Cannon, G. C. (2001) Carbon Cycling: The Prokaryotic Contribution. Curr. Opin. Microbiol. 4, 301-306.

(6) Bobik, T. A., Havemann, G. D., Busch, R. J., Williams, D. S., and Aldrich, H. C. (1999) The propanediol utilization (pdu) operon of Salmonella enterica serovar Typhimurium LT2 includes genes necessary for formation of polyhedral organelles involved in coenzyme $\mathrm{B}(12)$-dependent 1, 2-propanediol zyme B(12)-dependent 1, 2propanediol degradation. J. Bacteriol. 181, 5967-5975.

(7) Kofoid, E., Rappleye, C., Stojiljkovic, I., and Roth, J. (1999) The 17-gene ethanolamine (eut) operon of Salmonella typhimurium encodes five homologues of carboxysome shell proteins. J. Bacteriol. 181, 5317-5329.

(8) Petit, E., LaTouf, W. G., Coppi, M. V., Warnick, T. A., Currie, D., Romashko, I., Deshpande, S., Haas, K. N., Alvelo-Maurosa, J. G., Wardman, C., Schnell, D. J., Leschine, S. B., and Blanchard, J. L. (2013) Involvement of a bacterial microcompartment in the metabolism of fucose and rhamnose by Clostridium phytofermentans. PLoS One 8, e54337.

(9) Erbilgin, O., McDonald, K. L., and Kerfeld, C. A. (2014) Characterization of a Planctomycetal Organelle: a Novel Bacterial Microcompartment for the Aerobic Degradation of Plant Saccharides. Appl. Environ. Microbiol. 80, 2193-2205.

(10) Sampson, E. M., and Bobik, T. A. (2008) Microcompartments for B12-dependent 1,2-propanediol degradation provide protection from DNA and cellular damage by a reactive metabolic intermediate. J. Bacteriol. 190, 2966-2971.

(11) Tanaka, S., Kerfeld, C. A., Sawaya, M. R., Cai, F., Heinhorst, S., Cannon, G. C., and Yeates, T. O. (2008) Atomic-level models of the bacterial carboxysome shell. Science 319, 1083-1086.
(12) Sutter, M., Greber, B., Aussignargues, C., and Kerfeld, C. A. (2017) Assembly principles and structure of a 6.5-MDa bacterial microcompartment shell. Science 356, 1293-1297.

(13) Fan, C., Cheng, S., Sinha, S., and Bobik, T. A. (2012) Interactions between the termini of lumen enzymes and shell proteins mediate enzyme encapsulation into bacterial microcompartments. Proc. Natl. Acad. Sci. U. S. A. 109, 14995-15000.

(14) Jorda, J., Liu, Y., Bobik, T. A., and Yeates, T. O. (2015) Exploring Bacterial Organelle Interactomes: A Model of the ProteinProtein Interaction Network in the Pdu Microcompartment. PLoS Comput. Biol. 11, No. e1004067.

(15) Crowley, C. S., Cascio, D., Sawaya, M. R., Kopstein, J. S., Bobik, T. A., and Yeates, T. O. (2010) Structural Insight into the Mechanisms of Transport Across the Salmonella Enterica Pdu Microcompartment Shell. J. Biol. Chem. 285, 37838-37846.

(16) Kerfeld, C. A., Aussignargues, C., Zarzycki, J., Cai, F., and Sutter, M. (2018) Bacterial microcompartments. Nat. Rev. Microbiol. $16,277-290$

(17) Mangan, N. M., and Brenner, M. P. (2014) Systems analysis of the $\mathrm{CO} 2$ concentrating mechanism in cyanobacteria. eLife 3, No. e02043.

(18) Jakobson, C. M., Tullman-Ercek, D., Slininger, M. R., and Mangan, N. M. (2017) A systems-level model reveals that 1,2Propanediol utilization microcompartments enhance pathway flux through intermediate sequestration. PLoS Comput. Biol. 13, 069542.

(19) Lawrence, A. D., Frank, S., Newnham, S., Lee, M. J., Brown, I. R., Xue, W.-F., Rowe, M. L., Mulvihill, D. P., Prentice, M. B., Howard, M. J., and Warren, M. J. (2014) Solution Structure of a Bacterial Microcompartment Targeting Peptide and Its Application in the Construction of an Ethanol Bioreactor. ACS Synth. Biol. 3, 454-465.

(20) Yung, M. C., Bourguet, F. A., Carpenter, T. S., and Coleman, M. A. (2017) Re-directing bacterial microcompartment systems to enhance recombinant expression of lysis protein $\mathrm{E}$ from bacteriophage $\phi$ X174 in Escherichia coli. Microb. Cell Fact. 16, 71.

(21) Hussein, A. H., Lisowska, B. K., and Leak, D. J. (2015) The genus Geobacillus and their biotechnological potential. Adv. Appl. Microbiol. 92, 1-48.

(22) Nazina, T. N., Tourova, T. P., Poltaraus, A. B., Novikova, E. V., Grigoryan, A. A., Ivanova, A. E., Lysenko, A. M., Petrunyaka, V. V., Osipov, G. A., Belyaev, S. S., and Ivanov, M. V. (2001) Taxonomic study of aerobic thermophilic bacilli: descriptions of Geobacillus subterraneus gen. nov., sp. nov. and Geobacillus uzenensis sp. nov. from petroleum reservoirs and transfer of Bacillus stearothermophilus, Bacillus thermocatenulatus. Int. J. Syst. Evol. Microbiol. 51, 433-446.

(23) Aliyu, H., Lebre, P. H., Blom, J., Cowan, D. A., and de Maayer, P. (2016) Phylogenomic re-assessment of the thermophilic genus Geobacillus. Syst. Appl. Microbiol. 39, 527-533.

(24) Schallmey, M., Singh, A., and Ward, O. P. (2004) Developments in the use of Bacillus species for industrial production. Can. J. Microbiol. 50, 1-17.

(25) Liu, L., Liu, Y., Shin, H. D., Chen, R. R., Wang, N. S., Li, J., Du, G., and Chen, J. (2013) Developing Bacillus spp. as a cell factory for production of microbial enzymes and industrially important biochemicals in the context of systems and synthetic biology. Appl. Microbiol. Biotechnol. 97, 6113-6127.

(26) Finn, R. D., Mistry, J., Tate, J., Coggill, P., Heger, A., Pollington, J. E., Gavin, O. L., Gunasekaran, P., Ceric, G., Forslund, K., Holm, L., Sonnhammer, E. L. L., Eddy, S. R., and Bateman, A. (2010) The Pfam protein families database. Nucleic Acids Res. 38, D211-D222.

(27) Yeates, T. O., Crowley, C. S., and Tanaka, S. (2010) Bacterial Microcompartment Organelles: Protein Shell Structure and Evolution. Annu. Rev. Biophys. 39, 185-205.

(28) Jenuth, J. (1999) The NCBI: publicly available tools and resources on the Web. Methods Mol. Biol. 132, 301-312.

(29) Sheng, L., Zhang, Y., and Minton, N. P. (2016) Complete Genome Sequence of Geobacillus thermoglucosidasius NCIMB 11955, the Progenitor of a Bioethanol Production Strain. Genome Announc. 4, No. e1065-1016. 
(30) Taylor, M., Eley, K. L., Martin, S., Tuffin, M. I., Burton, S. G., and Cowan, D. A. (2009) Thermophilic ethanologenesis: future prospects for second-generation bioethanol production. Trends Biotechnol. 27, 398-405.

(31) Bartosiak-Jentys, J., Hussein, A. H., Lewis, C. J., and Leak, D. J. (2013) Modular system for assessment of glycosyl hydrolase secretion in Geobacillus thermoglucosidasius. Microbiology 159, 1267-1275.

(32) Brumm, P. J., Land, M. L., and Mead, D. A. (2015) Complete genome sequence of Geobacillus thermoglucosidasius C56-YS93, a novel biomass degrader isolated from obsidian hot spring in Yellowstone National Park. Stand. Genomic Sci. 10, 73.

(33) Bobik, T. A., Xu, Y., Jeter, R. M., Otto, K. E., and Roth, J. R. (1997) Propanediol utilization genes (pdu) of Salmonella typhimurium: three genes for the propanediol dehydratase. J. Bacteriol. 179, $6633-6639$.

(34) Trifunović, D., Schuchmann, K., and Müller, V. (2016) Ethylene glycol metabolism in the acetogen Acetobacterium woodii. J. Bacteriol. 198, 1058-1065.

(35) Palacios, S., Starai, V. J., and Escalante-Semerena, J. C. (2003) Propionyl Coenzyme A Is a Common Intermediate in the 1,2Propanediol and Propionate Catabolic Pathways Needed for Expression of the prpBCDE Operon during Growth of Salmonella enterica on 1,2-Propanediol. J. Bacteriol. 185, 2802-2810.

(36) Havemann, G. D., and Bobik, T. A. (2003) Protein content of polyhedral organelles involved in coenzyme B12-dependent degradation of 1,2-propanediol in Salmonella enterica serovar Typhimurium LT2. J. Bacteriol. 185, 5086-5095.

(37) Parsons, J. B., Dinesh, S. D., Deery, E., Leech, H. K., Brindley, A. A., Heldt, D., Frank, S., Smales, C. M., Lunsdorf, H., Rambach, A., Gass, M. H., Bleloch, A., McClean, K. J., Munro, A. W., Rigby, S. E. J., Warren, M. J., and Prentice, M. B. (2008) Biochemical and structural insights into bacterial organelle form and biogenesis. J. Biol. Chem. 283, 14366-14375.

(38) Xue, J., Murrieta, C. M., Rule, D. C., and Miller, K. W. (2008) Exogenous or 1-Rhamnose-Derived 1,2-Propanediol Is Metabolized via a pduD-Dependent Pathway in Listeria innocua. Appl. Environ. Microbiol. 74, 7073-7079.

(39) Chen, L., and Hatti-Kaul, R. (2017) Exploring Lactobacillus reuteri DSM20016 as a biocatalyst for transformation of longer chain 1,2-diols: Limits with microcompartment. PLoS One 12, No. e0185734.

(40) Parsons, J. B., Frank, S., Bhella, D., Liang, M., Prentice, M. B., Mulvihill, D. P., and Warren, M. J. (2010) Synthesis of Empty Bacterial Microcompartments, Directed Organelle Protein Incorporation, and Evidence of Filament-Associated Organelle Movement. Mol. Cell 38, 305-315.

(41) Sinha, S., Cheng, S., Fan, C., and Bobik, T. A. (2012) The PduM Protein Is a Structural Component of the Microcompartments Involved in Coenzyme B12-Dependent 1,2-Propanediol Degradation by Salmonella enterica. J. Bacteriol. 194, 1912-1918.

(42) Chowdhury, C., Sinha, S., Chun, S., Yeates, T. O., and Bobik, T. A. (2014) Diverse Bacterial Microcompartment Organelles. Microbiol. Mol. Biol. Rev. 78, 438-468.

(43) Fan, C., Cheng, S., Liu, Y., Escobar, C. M., Crowley, C. S., Jefferson, R. E., Yeates, T. O., and Bobik, T. A. (2010) Short Nterminal sequences package proteins into bacterial microcompartments. Proc. Natl. Acad. Sci. U. S. A. 107, 7509-7514.

(44) Scheinpflug, K., Wenzel, M., Krylova, O., Bandow, J. E., Dathe, M., and Strahl, H. (2017) Antimicrobial peptide cWFW kills by combining lipid phase separation with autolysis. Sci. Rep. 7, 44332.

(45) Matalon, E., Kaminker, I., Zimmermann, H., Eisenstein, M., Shai, Y., and Goldfarb, D. (2013) Topology of the trans-membrane peptide WALP23 in model membranes under negative mismatch conditions. J. Phys. Chem. B 117, 2280-2293.

(46) Pang, A., Frank, S., Brown, I., Warren, M. J., and Pickersgill, R. W. (2014) Structural insights into higher order assembly and function of the bacterial microcompartment protein PduA. J. Biol. Chem. 289, $22377-22384$.
(47) Noël, C. R., Cai, F., and Kerfeld, C. A. (2016) Purification and Characterization of Protein Nanotubes Assembled from a Single Bacterial Microcompartment Shell Subunit. Adv. Mater. Interfaces 3, 1500295.

(48) Mayer, M. J., Juodeikis, R., Brown, I. R., Frank, S., Palmer, D. J., Deery, E., Beal, D. M., Xue, W. F., and Warren, M. J. (2016) Effect of bio-engineering on size, shape, composition and rigidity of bacterial microcompartments. Sci. Rep. 6, 36899.

(49) Lehman, B. P., Chowdhury, C., and Bobik, T. A. (2017) The N Terminus of the PduB protein binds the protein shell of the Pdu microcompartment to its enzymatic core. J. Bacteriol. 199, No. e00785-00716.

(50) Fan, C., and Bobik, T. A. (2011) The N-Terminal Region of the Medium Subunit (PduD) Packages Adenosylcobalamin-Dependent Diol Dehydratase (PduCDE) into the Pdu Microcompartment. J. Bacteriol. 193, 5623-5628.

(51) Taylor, M. P., Esteban, C. D., and Leak, D. J. (2008) Development of a versatile shuttle vector for gene expression in Geobacillus spp. Plasmid 60, 45-52.

(52) Thompson, A., Studholme, D. J., Green, E. M., and Leak, D. J. (2008) Heterologous expression of pyruvate decarboxylase in Geobacillus thermoglucosidasius. Biotechnol. Lett. 30, 1359-1365.

(53) Buddrus, L., Andrews, E. S., Leak, D. J., Danson, M. J., Arcus, V. L., and Crennell, S. J. (2016) Crystal structure of pyruvate decarboxylase from Zymobacter palmae. Acta Crystallogr., Sect. F: Struct. Biol. Commun. 72, 700-706.

(54) Huber, I., Palmer, D. J., Ludwig, K. N., Brown, I., Warren, M. J., and Frunzke, J. (2017) Construction of recombinant pdu metabolosome shells for small molecule production in corynebacterium glutamicum. ACS Synth. Biol. 6, 2145-2156.

(55) Lee, M. J., Mantell, J., Brown, I. R., Fletcher, J. M., Verkade, P., Pickersgill, R. W., Woolfson, D. N., Frank, S., and Warren, M. J. (2018) De novo targeting to the cytoplasmic and luminal side of bacterial microcompartments. Nat. Commun. 9, 3413.

(56) Frank, S., Lawrence, A. D., Prentice, M. B., and Warren, M. J. (2013) Bacterial microcompartments moving into a synthetic biological world. J. Biotechnol. 163, 273-279.

(57) Lee, M. J., Brown, I. R., Juodeikis, R., Frank, S., and Warren, M. J. (2016) Employing bacterial microcompartment technology to engineer a shell-free enzyme-aggregate for enhanced 1,2-propanediol production in Escherichia coli. Metab. Eng. 36, 48-56.

(58) Nguyen, T. K., and Ueno, T. (2018) Engineering of protein assemblies within cells. Curr. Opin. Struct. Biol. 51, 1-8.

(59) Wagner, H. J., Capitain, C. C., Richter, K., Nessling, M., and Mampel, J. (2017) Engineering bacterial microcompartments with heterologous enzyme cargos. Eng. Life Sci. 17, 36-46.

(60) Plegaria, J. S., and Kerfeld, C. A. (2018) Engineering nanoreactors using bacterial microcompartment architectures. Curr. Opin. Biotechnol. 51, 1-7.

(61) Jung, Y. K., Kim, T. Y., Park, S. J., and Lee, S. Y. (2010) Metabolic engineering of Escherichia coli for the production of polylactic acid and its copolymers. Biotechnol. Bioeng. 105, 161-171.

(62) Peralta-Yahya, P. P., Zhang, F., del Cardayre, S. B., and Keasling, J. D. (2012) Microbial engineering for the production of advanced biofuels. Nature 488, 320-328.

(63) Young, E. J., Burton, R., Mahalik, J. P., Sumpter, B. G., FuentesCabrera, M., Kerfeld, C. A., and Ducat, D. C. (2017) Engineering the Bacterial Microcompartment Domain for Molecular Scaffolding Applications. Front. Microbiol. 8, 1441.

(64) Punta, M., Coggill, P. C., Eberhardt, R. Y., Mistry, J., Tate, J., Boursnell, C., Pang, N., Forslund, K., Ceric, G., Clements, J., Heger, A., Holm, L., Sonnhammer, E. L. L., Eddy, S. R., Bateman, A., and Finn, R. D. (2012) The Pfam protein families database. Nucleic Acids Res. 40, D290-301.

(65) Altschul, S. F., Madden, T. L., Schäffer, A. A., Zhang, J., Zhang, Z., Miller, W., and Lipman, D. J. (1997) Gapped BLAST and PSIBLAST: a new generation of protein database search programs. Nucleic Acids Res. 25, 3389-3402. 
(66) Naville, M., Ghuillot-Gaudeffroy, A., Marchais, A., and Gautheret, D. (2011) ARNold: A web tool for the prediction of Rho-independent transcription terminators. RNA Biol. 8, 11-13.

(67) Katoh, K., and Standley, D. M. (2014) MAFFT: iterative refinement and additional methods. Methods Mol. Biol. 1079, 131146.

(68) Jones, D. T., Taylor, W. R., and Thornton, J. M. (1992) The rapid generation of mutation data matrices from protein sequences. Bioinformatics 8, 275-282.

(69) Letunic, L., and Bork, P. (2016) Interactive tree of life (iTOL) v3: an online tool for the display and annotation of phylogenetic and other trees. Nucleic Acids Res. 44, W242-W245.

(70) Bryksin, A. V., and Matsumura, I. (2010) Overlap extension PCR cloning: a simple and reliable way to create recombinant plasmids. BioTechniques 48, 463-465.

(71) Miyazaki, K. (2011) MEGAWHOP cloning: a method of creating random mutagenesis libraries via megaprimer PCR of whole plasmids. Methods Enzymol. 498, 399-406.

(72) Sambrook, J., Fritsch, E. F., and Maniatis, T. (1989) Molecular Cloning: A Laboratory Manual, Cold Spring Harbor Laboratory Press, New York.

(73) Hamoen, L. W., Smits, W. K., de Jong, A., Holsappel, S., and Kuipers, O. P. (2002) Improving the predictive value of the competence transcription factor (ComK) binding site in Bacillus subtilis using a genomic approach. Nucleic Acids Res. 30, 5517-5528.

(74) Daniel, R. A., Haiech, J., Denizot, F., and Errington, J. (1997) Isolation and characterization of the lacA gene encoding betagalactosidase in Bacillus subtilis and a regulator gene, lacR. J. Bacteriol. 179, 5636-5638.

(75) Santos-Beneit, F., Roberts, D. M., Cantlay, S., McCormick, J. R., and Errington, J. (2017) A mechanism for FtsZ-independent proliferation in Streptomyces. Nat. Commun. 8, 1378.

(76) Meyvis, T., de Smedt, S. C., van Oostveldt, P., and Demeester, J. (1999) Fluorescence Recovery After Photobleaching: A Versatile Tool for Mobility and Interaction Measurements in Pharmaceutical Research. Pharm. Res. 16, 1153-1162.

(77) Schneider, C. A., Rasband, W. S., and Eliceiri, K. W. (2012) NIH Image to ImageJ: 25 years of image analysis. Nat. Methods 9, 671-675. 